\title{
Combined ultrasonic and photoacoustic system for deep tissue imaging
}

Chulhong Kim, Todd N. Erpelding, Ladislav Jankovic, Lihong V. Wang

Chulhong Kim, Todd N. Erpelding, Ladislav Jankovic, Lihong V. Wang, "Combined ultrasonic and photoacoustic system for deep tissue imaging," Proc. SPIE 7899, Photons Plus Ultrasound: Imaging and Sensing 2011, 789935 (22 February 2011); doi: 10.1117/12.874914

SPIE. Event: SPIE BiOS, 2011, San Francisco, California, United States 


\title{
Combined Ultrasonic and Photoacoustic System for Deep Tissue Imaging
}

\author{
Chulhong Kim ${ }^{1}$, Todd N. Erpelding ${ }^{2}$, Ladislav Jankovic ${ }^{2}$, and Lihong V. Wang ${ }^{1 *}$ \\ ${ }^{1}$ Optical Imaging Laboratory, Department of Biomedical Engineering, Washington University in St. \\ Louis, Campus Box 1097, One Brookings Dr. St. Louis, Missouri, 63130 \\ ${ }^{2}$ Philips Research North America, 345 Scarborough Road, Briarcliff Manor, NY 10510
}

\begin{abstract}
A combined ultrasonic and photoacoustic imaging system is presented that is capable of deep tissue imaging. The system consists of a modified clinical ultrasound array system and tunable dye laser pumped by a Nd:YAG laser. The system is designed for noninvasive detection of sentinel lymph nodes and guidance of needle biopsies for axillary lymph node staging in breast cancer patients. Using a fraction of the American National Standards Institute (ANSI) safety limit, photoacoustic imaging of methylene blue achieved penetration depths of greater than $5 \mathrm{~cm}$ in chicken breast tissue. Photoacoustic imaging sensitivity was measured by varying the concentration of methylene blue dye placed at a depth of $3 \mathrm{~cm}$ within surrounding chicken breast tissue. Signal-to-noise ratio, noise equivalent sensitivity, and axial spatial resolution were quantified versus depth based on in vivo and chicken breast tissue experiments. The system has been demonstrated in vivo for detecting sentinel lymph nodes in rats following intradermal injection of methylene blue. These results highlight the clinical potential of photoacoustic image-guided identification and needle biopsy of sentinel lymph nodes for axillary staging in breast cancer patients.
\end{abstract}

Keywords: Photoacoustic imaging, sentinel lymph node biopsy, image-guided needle biopsy

\section{INTRODUCTION}

To minimize post-operative complications of axillary lymph node dissection (ALND), sentinel lymph node biopsy (SLNB), a biopsy of the first draining lymph node in breast cancer region, is the standard process for staging breast cancer patients. ${ }^{1}$ Although current SLNB using methylene blue dye and radioactive materials have an identification rate of $90-95 \%$ and a sensitivity of $88-95 \%$, this method is ionizing and invasive. Moreover, the false negative rate is still 5$10 \%{ }^{2,3}$ In addition, due to the surgical removal of the sentinel lymph node, the SLNB still poses postoperative complications like ALND, including lymphedema, seroma formation, impairment of shoulder movement, and postoperative pain. ${ }^{4}$ As a minimally invasive method to reduce abovementioned complications, ultrasound-guided fine needle aspiration biopsy (FNAB) has been tested clinically. ${ }^{5}$ Yet, ultrasound imaging itself cannot distinguish the sentinel lymph node from other lymph nodes. Therefore, there is still a pressing need to develop an accurate, reliable, non-ionizing, and non-invasive detection method for lymph node assessment.

Photoacoustic (PA) imaging is an optical imaging modality that can provide strong optical contrast and high ultrasonic resolution. ${ }^{6,7}$ The image resolution can be tuned with ultrasonic frequency within the reach of diffusive photons. Moreover, the pixel number, defined as the ratio of the maximum imaging depth to the spatial resolution, exceeds 100 for PA imaging. In biological tissues, the imaging depth can be up to a few centimeters deep. Methylene blue, indocyanine green, and gold nanoparticles have been applied as lymph node tracers in rats in vivo using PA imaging. ${ }^{8-12}$

We previously reported in vivo PA and US mapping of rat sentinel lymph nodes (SLNs) using a hand-held probe adapted from a clinical US imaging system. ${ }^{10-12}$ In this proceedings paper, we quantified signal-to-noise ratio (SNR), axial spatial resolution, and sensitivity of the hand-held PA probe by measuring chicken breast tissue ex vivo and in vivo rat SLNs.

\footnotetext{
* Corresponding author: lhwang@biomed.wustl.edu
} 


\section{METHODS AND MATERIALS}

The combined PA and US imaging probe was developed around a commercial clinical ultrasound scanner (iU22, Philips Healthcare) and has been described previously ${ }^{11,12}$. In brief, raw per-channel PA and US data were both acquired through the modified channel board architecture and transferred to a custom-made data acquisition computer for display and post-processing. Laser firing was synchronized with the data acquisition system. A tunable dye laser (PrecisionScan-P, Sirah), pumped by a Q-switched Nd:YAG laser (PRO-350-10, Newport), produced 6.5-ns laser pulses at a repetition rate of $10 \mathrm{~Hz}$, which illuminated tissue via bifurcated fiber bundles (CB18043, Fiberguide). Generated PA waves were detected by a linear US array probe (L8-4, Philips Healthcare) with a nominal bandwidth of 4-8 MHz. An optical wavelength of $650 \mathrm{~nm}$, close to the peak optical absorption wavelength of methylene blue (667 $\mathrm{nm}$ ), was utilized. Light fluence on the surface was $\sim 3 \mathrm{~mJ} / \mathrm{cm}^{2}$, only $1 / 7$ of the ANSI safety limit $\left(20 \mathrm{~mJ} / \mathrm{cm}^{2}\right){ }^{12}$ PA images were reconstructed using a Fourier beam-forming algorithm and displayed at $\sim 1$ frame per second (fps). The PA signals were averaged 20 times.

A plastic tube $(7 \mathrm{~mm}$ in diameter $\times 25 \mathrm{~mm}$ in length) filled with methylene blue was embedded in chicken breast tissues. Methylene blue is routinely used in clinical practice for SLNB. The concentration of methylene blue was $\sim 30$ $\mathrm{mM}$. By layering additional chicken breast tissue, we increased the imaging depth, as measured by US imaging.

Animal care and use was in accordance with approved guidelines at Washington University in St. Louis. We imaged Sprague Dawley rats $(\sim 200 \mathrm{~g})$ under anesthesia after injection of a mixture of ketamine $(80 \mathrm{mg} / \mathrm{kg})$ and xylazine $(8$ $\mathrm{mg} / \mathrm{kg}$ ). For in vivo imaging, we placed chicken breast tissue atop the rats to increase the imaging depth. A control PA image was acquired before intradermal injection of methylene blue into the left forepaw. After injection of methylene blue $(0.1 \mathrm{~mL}, 30 \mathrm{mM})$, a series of PA images were acquired to identify SLNs enhanced by methylene blue.

\section{RESULTS AND DISCUSSION}

Figure 1 shows the tube filled with methylene, positioned $1.9 \mathrm{~cm}$ deep in chicken tissues. The PA image clearly maps the top and bottom boundaries of the tube, and the co-registered PA-US image confirmed the location of the tube in chicken tissues.

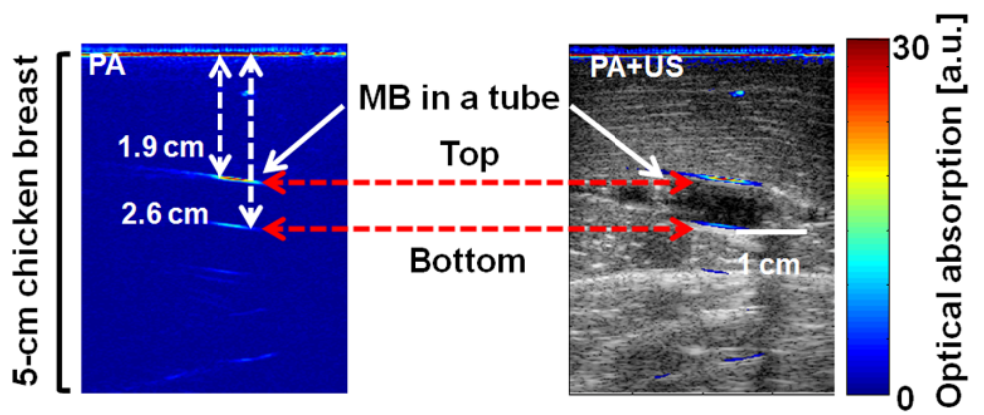

Figure 1: Photoacoustic (PA) and ultrasound (US) imaging of methylene blue (MB) in biological tissues. The tube was filled with MB as positioned within a stack of chicken breast tissue. Overlaid PA (pseudocolor) and US (grayscale) images shows the tube containing $\mathrm{MB}$ at depths of $1.9 \mathrm{~cm}$. Pseudocolor represents the optical absorption in laboratory units.

The SNR and axial spatial resolution of the PA imaging system were quantified by embedding a plastic tube filled with methylene blue dye at various depths within chicken breast tissue. Figure 2 is a plot of the SNR as a function of the depth of the tube. The SNR was defined as the mean of PA signal amplitudes obtained from the tube divided by the standard deviation of the background signals. The SNR decreased linearly with increasing depth. The SNRs at 1.9 and $4.55 \mathrm{~cm}$ are 108 and 10, respectively. The measured penetration depth for $1 / e$ decay in chicken breast tissue is $\sim 1.1 \mathrm{~cm}$, which matches well with the value reported in the literature $(\sim 1.13 \mathrm{~cm})$ at an optical wavelength of $650 \mathrm{~nm}$. ${ }^{13}$ Note that the $4.5-\mathrm{cm}$ penetration depth was achieved with a laser fluence of $3 \mathrm{~mJ} / \mathrm{cm}^{2}$ (only $1 / 7$ of the ANSI safety limit). By 
using a laser fluence of $20 \mathrm{~mJ} / \mathrm{cm}^{2}$ (the ANSI safety limit), the penetration depth can theoretically be increased to $\sim 7$ $\mathrm{cm}$.

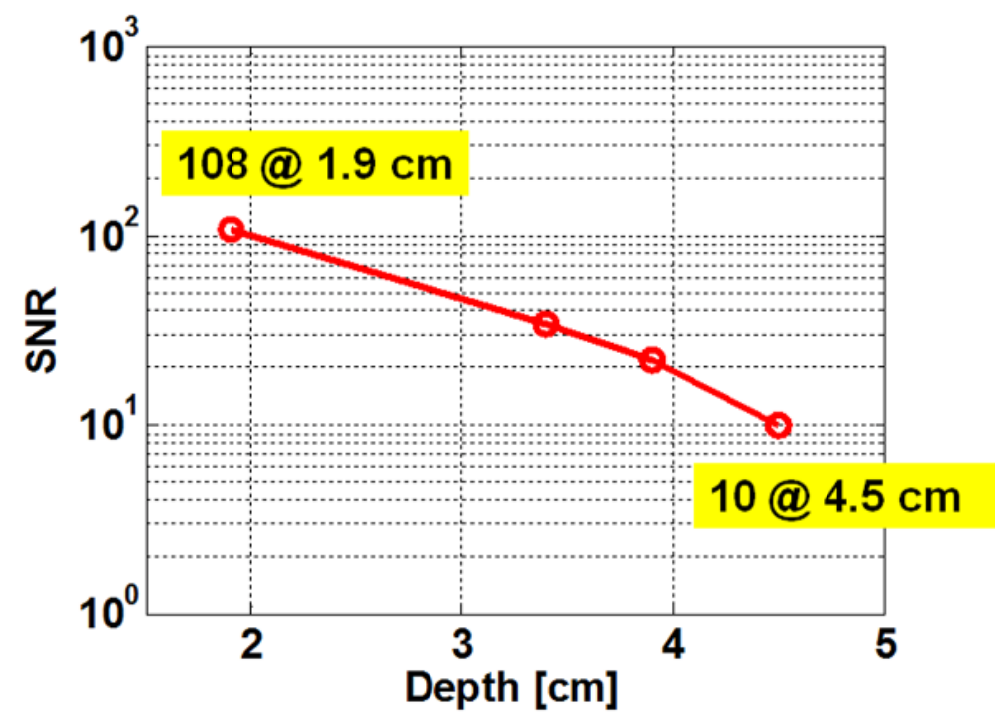

Figure 2: Plot of SNR as a function of depth of the tube containing $30 \mathrm{mM}$ of methylene blue.

The noise-equivalent sensitivity, defined as the ratio between dye concentration and SNR, was plotted as a function of depth in Figure 3. The noise-equivalent sensitivity is $\sim 0.27$ and $3 \mathrm{mM}$ at a depth of 1.9 and $4.5 \mathrm{~cm}$, respectively.

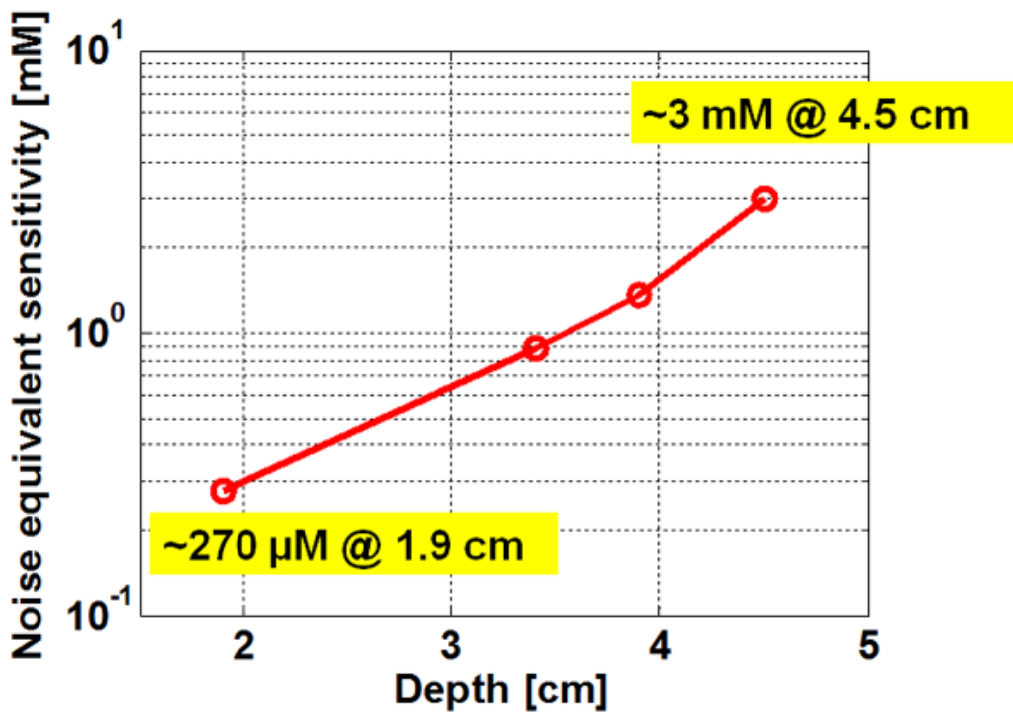

Figure 3: Plot of noise equivalent sensitivity as a function of depth of the tube containing $\sim 30 \mathrm{mM}$ of methylene blue.

The axial spatial resolution at various imaging depths in chicken breast tissue was measured as the full width at half maximum (FWHM) of the 1D profile taken across the tube boundary (Fig. 4). The axial resolutions measured from the tube boundaries at all imaging depths were $\sim 400 \mu \mathrm{m}$, which was close to the theoretical axial resolution ( $\sim 385 \mu \mathrm{m}$, for a nominal bandwidth of 4-8 MHz). 


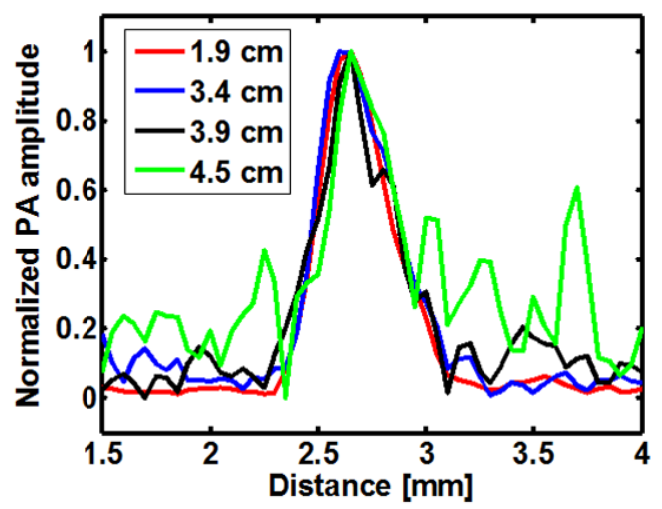

Figure 4: 1D axial profiles taken across the boundary of the tube containing methylene blue $(\sim 30 \mathrm{mM})$ provide an estimate of the FWHM as a function of depth.

We imaged the left axillary region in a rat. Soon after injection of methylene blue, the SLN was identified photoacoustically. We overlaid additional chicken breast tissue atop the targets to increase the imaging depth $(\sim 3.8 \mathrm{~cm})$. The structural US image (gray color) and functional PA image (pseudocolor, methylene blue uptake in the SLN) are overlaid in Fig. 5 (left). The SNR was plotted as a function of the imaging depth (Fig. 5, right). The measured penetration depth for 1/e decay is $1.06 \mathrm{~cm}$, which matches well with the above mentioned ex vivo 1/e penetration depth $(1.13 \mathrm{~cm})$. Postmortem dissection of rat sentinel lymph nodes confirmed uptake of methylene blue dye.

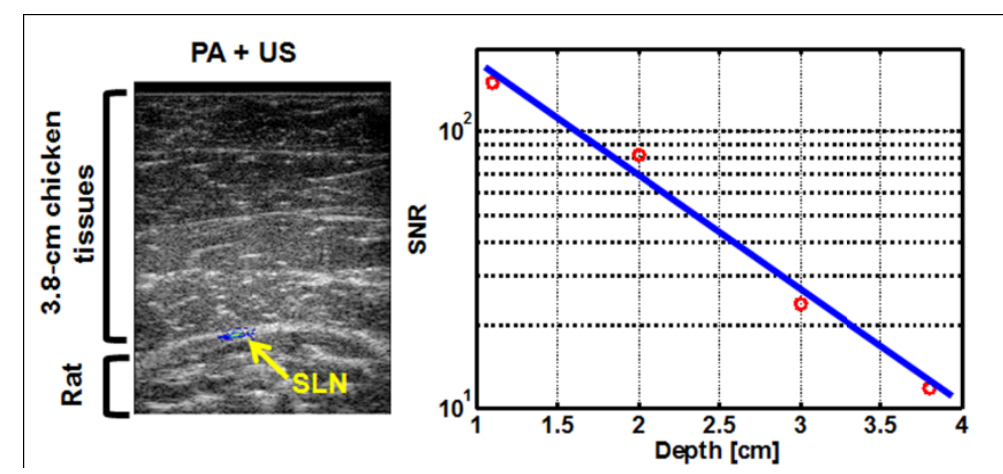

Figure 5: In vivo photoacoustic (PA) mapping of rat sentinel lymph nodes (SLNs) and ultrasound (US) mapping of surrounding anatomical structure (left). The depth of the SLN from the imaging surface was $\sim 3.8 \mathrm{~cm}$. Pseudocolor represents the optical absorption in laboratory units. The right figure shows a plot of PA SNR as a function of depth of the SLN. Additional layers of chicken breast were placed atop the rat to increase the imaging depth.

\section{CONCLUSIONS}

In conclusion, the capabilities - including SNR, axial resolution, and sensitivity - of the hand-held PA imaging probe were quantified in biological tissues. The maximum penetration depth reached $\sim 4.5 \mathrm{~cm}$ with an SNR of 10 . The noiseequivalent sensitivities were 0.27 and $3 \mathrm{mM}$ ex vivo at 1.9 and $4.5 \mathrm{~cm}$ deep, respectively. The axial resolution was maintained over the imaging depths observed. Further, the estimated optical property (effective attenuation coefficient) of chicken breast tissue matched well with previously reported values. Moreover, in vivo PA and US mapping of rat SLNs at an imaging depth of $\sim 3.8 \mathrm{~cm}$ was successfully accomplished following methylene blue injection.

\section{ACKNOWLEDGEMENT}

This work was supported in part by grants from National Institutes of Health (R01 EB000712, R01 EB008085, R01 NS46214, and U54 CA136398 -- the Network for Translational Research -- to L.V.W.). L.V.W. has a financial interest in Endra, Inc., which, however, did not support this work. 


\section{REFERENCES}

[1] H. Kobayashi, S. Kawamoto, Y. Sakai, et al., "Lymphatic drainage imaging of breast cancer in mice by micromagnetic resonance lymphangiography using a nano-size paramagnetic contrast agent," J. Natl. Cancer. I. 96(9), 703708 (2004).

[2] K. M. McMasters, T. M. Tuttle, D. J. Carlson, et al., "Sentinel lymph node biopsy for breast cancer: a suitable alternative to routine axillary dissection in multi-institutional practice when optimal technique is used," J. Clin. Oncol., 18(13), 2560-2566 (2000).

[3] O. A. Ung, "Australasian experience and trials in sentinel lymph node biopsy: the RACS SNAC trial," Asian. J. Surg. 27(4), 284-290 (2004).

[4] A. D. Purushotham, S. Upponi, M. B. Klevesath, et al., "Morbidity after sentinel lymph node biopsy in primary breast cancer: Results from a randomized controlled trial," J. Clin. Oncol. 23(19), 4312-4321 (2005).

[5] S. Krishnamurthy, N. Sneige, D. G. Bedi, et al., "Role of ultrasound-guided fine-needle aspiration of indeterminate and suspicious axillary lymph nodes in the initial staging of breast carcinoma," Cancer 95(5), 982-988 (2002).

[6] C. Kim, C. Favazza and L. V. Wang, "In vivo photoacoustic tomography of chemicals: high-resolution functional and molecular optical imaging at new depths," Chem. Rev. 110(5), 2756-2782(2010).

[7] H. F. Zhang, K. Maslov, G. Stoica, et al., "Functional photoacoustic microscopy for high-resolution and noninvasive in vivo imaging," Nat. Biotechnol. 24(7), 848-851 (2006).

[8] K. H. Song, C. Kim, C. M. Cobley, et al., "Near-infrared gold nanocages as a new class of tracers for photoacoustic sentinel lymph node mapping on a rat model," Nano Lett. 9(1), 183-188 (2009).

[9] C. Kim, K. H. Song, F. Gao, and L. V. Wang, " Sentinel lymph nodes and lymphatic vessels: noninvasive dualmodality in vivo mapping by using indocyanine green in rats - volumetric spectroscopic photoacoustic imaging and planar fluorescence imaging,” Radiology 255, 442-450 (2010).

[10] T. N. Erpelding, C. Kim, M. Pramanik, et al., "Sentinel lymph nodes in the rat: noninvasive photoacoustic and US imaging with a clinical US system," Radiology 256(1), 102-110 (2010).

[11] C. Kim, T. N. Erpelding, K. Maslov, et al., "Handheld array-based photoacoustic probe for guiding needle biopsy of sentinel lymph nodes," J. Biomed. Opt. 15(4), 046010 (2010).

[12] C. Kim, T. N. Erpelding, L. Jankovic, et al., "Deeply penetrating in vivo photoacoustic imaging using a clinical ultrasound array system," Biomed. Opt. Express, 1(1), 278-284 (2010).

[13] G. Marquez, G., L. V. Wang, P. S. Schwartz, et al., "Anisotropy in the absorption and scattering spectra of chicken breast tissue," Appl. Opt. 37(4), 798-804 (1998). 